\title{
Direct imaging of massive extrasolar planets
}

\author{
Paul Kalas ${ }^{1}$ \\ ${ }^{1}$ Astronomy Department, University of California \\ Berkeley, CA 94720, USA \\ email: kalas@berkeley.edul
}

\begin{abstract}
The direct detection of an extrasolar planet can provide accurate measurements of its orbit, mass and composition, greatly improving our understanding of how planets form and evolve. Recent advances in ground-based and space-based imaging techniques have now produced the first direct images of extrasolar planets. Typically these are many-Jupiter-mass planets on wide orbits. Direct imaging therefore probes the outer architecture of planetary systems and it is highly complementary to other techniques sensitive to inner architectures. This brief review summarizes the properties of the currently imaged exoplanets, provides an update on the orbit of Fomalhaut $b$, and highlights the emerging phenomenon of circumplanetary disks.
\end{abstract}

Keywords. planetary systems, planets and satellites: rings, accretion, accretion disks, interplanetary medium, stars: individual (Fomalhaut)

\section{Introduction}

The direct imaging of extrasolar planets has been one of the late bloomers among the various detection techniques. Direct imaging could of course refer to any method that measures photons that interact with an extrasolar planets. Therefore the thermal infrared light curves that give secondary eclipse light curves, primary eclipse spectroscopy, or some periodic polarization signal might be considered a sort of image for an extrasolar planet (e.g., see Seager \& Deming 2010 for a review).

This review covers the scientific findings from recent observations where the planet signal is spatially resolved from the star. Readers interested in understanding the technical problems and the current state-of-the-art may refer to reviews by Duchene (2008) and Oppenheimer \& Hinkley (2009).

Table 1 gives a snapshot of the currently observed extrasolar planets arranged in order of increasing heliocentric distance (column 3). I will briefly discuss each of the topics in the table columns, provide an update on Fomalhaut b, and touch on several aspects of circumplanetary material. My approach is to make this information memorable for astronomy students, but with enough detail to make it valuable for more senior scientists.

\section{Host and Spectral Type}

Probably the most common question asked by scientists listening to talks on directly imaged planets is the following: "Has anyone obtained RV data on these stars?". Columns 1 and 2 in Table 1 provide the answer.

Column 1 is labeled "host" rather than "host star" because several of the currently detected planets appear to be physically associated with either a brown dwarf (2M1207) or a pre-main sequence object (GQ Lup, 1RXJ1609, CT Cha). RV detected planets are for the most part limited to bright, stable, FGKM main-sequence stars, whereas the brown 
Table 1. Overview of directly imaged extrasolar planets

\begin{tabular}{|c|c|c|c|c|c|c|c|}
\hline Host & $|\mathrm{SpT}|$ & $\left|\begin{array}{c}\text { Distance } \\
{[\mathrm{pc}]}\end{array}\right|$ & Planet & $\mid \begin{array}{c}\text { Separation } \\
{[\mathrm{AU}, \text { projected }]}\end{array}$ & $\begin{array}{c}\text { Mass } \\
{\left[M_{J}\right]}\end{array}$ & $\begin{array}{l}\text { Age } \\
{[\mathrm{Myr}]}\end{array}$ & Reference \\
\hline | Fomalhaut & $|\mathrm{A} 4 \mathrm{~V}|$ & 7.69 & $b$ & 100 & $<3$ & $100-400$ & Kalas et al. (2008) | \\
\hline$\beta \mathrm{Pic}$ & $|\mathrm{A} 5 \mathrm{~V}|$ & 19.3 & $b$ & 8 & $7-11$ & $8-20$ & Lagrange et al. (2009)| \\
\hline HR 8799 & A $5 \mathrm{~V}$ & $39.4 \pm 1.0$ & $\begin{array}{l}b \\
c \\
d \\
e\end{array}$ & $\begin{array}{c}68 \\
38 \\
24 \\
14.5\end{array}$ & $\begin{array}{l}4-10 \\
7-13 \\
7-13 \\
5-13\end{array}$ & $30-160$ & $\begin{array}{r}\text { Marois et al. }(2008) \\
\prime \prime \\
\text { Marois et al. }(2010)\end{array}$ \\
\hline $\mathrm{AB} \mathrm{Pic}$ & $|\mathrm{K} 2 \mathrm{~V}|$ & $|45.5 \pm 1.6|$ & $b$ & 260 & $|11-16|$ & 30 & Chauvin et al. (2005) \\
\hline $2 \mathrm{M} 1207$ & $\mathrm{~L} 2$ & $|52.4 \pm 1.1|$ & $b$ & 41 & $2-10$ & $5-12$ & Chauvin et al. (2004) \\
\hline GQ Lup & $\mathrm{K} 7$ & $156 \pm 50$ & $b$ & 100 & $4-39$ & $<2$ & Neuhauser et al. (2005)| \\
\hline 1RXJ1609 & $\mathrm{K} 7$ & $145 \pm 20$ & $b$ & 330 & $6-11$ & $4-6$ & Lafreniere et al. (2010) \\
\hline CT Cha & K7 & $165 \pm 30$ & $b$ & 440 & $|11-23|$ & $<4$ & Schmidt et al. (2008) \\
\hline
\end{tabular}

Notes: For the significance of non-detections, see, e.g., Lafreniere et al. 2007; Nielsen \& Close 2010; Chauvin et al. 2010. Essentially for every host target in Table 1, at least ten more were imaged with a null result.

dwarf and pre-main sequence hosts are both faint and variable. For example, 2M1207b is a common proper motion companion to 2MASSW J1207334-393254 (2M1207A), which is a mid-L dwarf with $M \sim 25 M_{J}$. With $m_{V}=20.2 \mathrm{mag}$ (Ducourant et al. 2008), 2M1207A is not amenable to RV observations.

The brighter, main-sequence host stars Fomalhaut, Beta Pic and HR 8799 are all A stars $\left(M \sim 1.7-2.9 M_{\odot}\right)$. RV techniques are unsuccessful because stellar lines are fewer, shallower and rotationally broadened (100 - $200 \mathrm{~km} \mathrm{~s}^{-1}$; Galland et al. 2005). However, a separate sample of older, "retired" A stars (i.e., subgiants) have slower rotation and more lines, permitting an RV-derived estimate that the exojupiter occurrence rate is relatively high $(\sim 26 \%)$ for intermediate mass stars (Bowler et al. 2010).

In some cases, exoplanets may have been imaged as free floaters in young clusters, and the "host" might be the cluster name. Specifically, the 3 Myr-old $\sigma$ Orionis cluster at $\sim 400$ pc may contain several planet-mass objects (Zapatero Osorio et al. 2000), and other young clusters such as IC 348 (Luhman et al. 2005) and the Trapezium (Lucas et al. 2006) are now targets for finding planet-mass objects via direct imaging and spectroscopy. The free floaters are not represented in Table 1.

These findings of free-floating planets and planets associated with brown dwarfs challenge the notion that planets necessarily form in circumstellar disks in a manner completely different from stars. Moreover, the large separations between the planets and host stars rule out in situ formation by core-accretion in a circumstellar disk, with gravitational instability as a more viable mechanism. For a more complete analysis, see recent work by Dodson-Robinson et al. (2009), Nero \& Bjorkman (2009), \& Kratter et al. (2010), to name a few. A different class of models assumes a birth site closer to the star with subsequent dynamical transport outward (e.g., Rasio \& Ford 1996; Veras et al. 2009; Raymond et al. 2010).

\section{Distance and Separation}

A near simultaneous glance at the Distance and Age columns in Table 1 reveals that direct imaging searches for exoplanets have been successful for the very young $\left(<10^{6}\right.$ yr) star-forming environments at $>140 \mathrm{pc}$, and for the somewhat older $\left(10^{6}-10^{7} \mathrm{yr}\right)$ main sequence stars residing within the local bubble $(<100 \mathrm{pc})$. The next generation 
of extreme adaptive optics systems such as the Gemini Planet Imager (GPI; Macintosh et al. 2008) sample the host star wavefront at rates exceeding $2 \mathrm{kHz}$ and target stars must therefore have $\mathrm{m}_{I}<9 \mathrm{mag}$ ( $I$-band is centered near $\left.0.8 \mu \mathrm{m}\right)$. Thus, future ground based imaging experiments will have the greatest impact in discovering new planets orbiting main sequence stars within the local bubble.

The fundamental breakthrough of future imaging experiments is that the inner working angle (IWA) will be reduced (improved) to a few $\lambda / \mathrm{D}$ radius from the star. The IWA specifies the smallest radial distance from a star where a planet-mass object could be detectable. Currently, the $\beta$ Pic $b$ and HR $8799 e$ detections represent the smallest achievable IWA's. A near simultaneous glance at the Distance and Separation columns, and dividing the latter by the former, shows that both $\beta$ Pic $b$ and HR $8799 e$ are detected at IWA $\sim 0.4^{\prime \prime}$ radius. The goal for GPI is IWA $\sim 3.5 \lambda / \mathrm{D}$. Since the Gemini $8-\mathrm{m}$ telescope has an effective aperture of $\mathrm{D}=7.77 \mathrm{~m}$, then IWA $=0.15^{\prime \prime}$ at $1.6 \mu \mathrm{m}(H$-band $)$. Of course the star-to-planet contrast achieved beyond $0.15^{\prime \prime}$ radius is the other significant metric of planet imaging experiments. The various components of GPI are designed to achieve a goal of $\Delta H>15$ mag.

Table 1 gives the host-to-exoplanet projected separations $(\rho)$, and not the exoplanet deprojected separations $(r)$ in a stellocentric cylindrical or spherical coordinate system, nor the semi-major axes, $(a)$. Moreover, the projected separations are variable due to the exoplanet orbital motion. For $\beta$ Pic and Fomalhaut, images of light scattering from circumstellar disk grains give line-of-sight disk inclinations of $i_{d} \sim 90^{\circ}$ (edge-on) and $i_{d} \sim$ $24^{\circ}$, respectively (Smith \& Terrile 1984; Kalas et al. 2005). Assuming circular $(e=0.0)$ and coplanar exoplanet orbits, the host-exoplanet separations correspond to $r \sim 8 \mathrm{AU}$ for $\beta$ Pic $b$, and $r \sim 119$ AU for Fomalhaut $b$. However, neither assumption is well-tested for any of the exoplanets in Table 1, largely because only a few epochs of astrometry are available. For example, adopting the assumption that Fomalhaut $b$ lies in the belt plane, if $e=0.12$, as deduced from the measured stellocentric belt offset (Kalas et al. 2005), then $a \sim 115$ AU.

The conversion from apparent separation to semi-major axis is particularly important for multi-planet systems such as HR 8799, where the origin and evolution of the system hinges on dynamical stability analysis (e.g., Fabrycky \& Murray-Clay 2010). Not shown in Table 1 are the position angle (PA) of each exoplanet relative to the star, and the PA for the semi-major axis of each debris disk, which are required for the deprojection calculation (here the semi-major axis does not refer to an orbital element; instead it refers to the apparent elliptical morphology of an inclined circle). HR 8799 is surrounded by a debris disk that is resolved at $70 \mu \mathrm{m}$ with the Spitzer Space Telescope, indicating $i_{d}<25^{\circ}$ (Su et al. 2009). If we adopt $i_{d}=20^{\circ}$ and put the major axis along the direction from the star to HR 8799b, then for the $b$ component we have $\rho=r$, but for HR $8799 c$, which is approximately orthogonal to $b$, the non-face-on inclination translates to $\rho \sim$ $r / \cos \left(20^{\circ}\right)$. In other words, $r$ is $6 \%$ greater than the projected separation $\rho$. Reidemeister et al. (2009) consider the entire range of position angles, finding that with $i_{d}=20^{\circ}$, position angles of approximately $0^{\circ}-90^{\circ}$ give stable configurations over the age of the system.

From Table 1, $\beta$ Pic $b$ is at the separation most "Jupiter-like" as it resides near the ice-line of the system. Since the inclination and position angle of the disk are well-constrained, it is unlikely that the semi-major axis of $\beta$ Pic $b$ will be shown to be more than 1 AU different from the current estimate of 8 AU. The corresponding $\sim 16$ year orbital period means that in their lifetime, the majority of readers will witness a full orbit of $\beta$ Pic $b$, whereas for Fomalhaut $b$ readers need to wait eight centuries. 


\section{Planet Mass and Age}

Stellar age uncertainties may be the most important factor in determining whether or not an object is a planet or a brown dwarf $\left(M>13 M_{J}\right)$. In the literature one will typically find a paper announcing the discovery of an extrasolar planet through direct imaging, and then at least one follow-up paper discussing the age of the star and the implications for planet masses. For example, Marois et al. (2008) estimate the age of HR 8799 as $<160 \mathrm{Myr}$, which means that all three planets in their manuscript have $M \leqslant 13 M_{J}$. Moya et al. (2010) then describe the age uncertainty in greater detail, and present evidence from astroseismology that supports a likely age near 1 Gyr, implying that the HR 8799 objects have brown dwarf masses. Moro-Martin et al. (2010) respond that the system is dynamically stable only for $\leqslant 150 \mathrm{Myr}$, even if the masses are in the brown dwarf regime, and this timescale is a hard constraint. A new dynamical analysis that adds the fourth planet, HR 8799e, suggests that the planet masses must be relatively small, and therefore from the measured luminosities and theoretical cooling curves, the system is younger than 60 Myr (Marois et al. 2010).

The analytic estimate for the luminosity evolution of a brown dwarf gives $L \propto M^{2.6}$ $t^{-1.3}$ (Burrows \& Liebert 1993). Numerical calculations extending down to Jupiter mass planets give $L \propto M^{1.9} t^{-1.1}$ (Burrows et al. 2003; Fortney et al. 2008). A factor of two uncertainty in age corresponds to a $50 \%$ uncertainty in mass. Of course there are many more variables that matter, such as the assumptions of chemistry, metallicity, clouds, etc. One significant difference concerns the assumptions of planet formation, as illustrated in Marley et al. (2007) and Fortney et al. (2008). The scalings above apply to the hot-start planet formation model, but in a cold-start model accreting gas passes through a shock that quickly radiates a large fraction of the energy early in the evolution of the planet. The young exojupiter thereby acquires relatively cool gas, and therefore a low luminosity does not necessarily mean a less massive planet at a given age.

The scientific importance of direct imaging should therefore be obvious. This is the method by which we can empirically anchor theories of planet formation and evolution. Planet masses with direct imaging can be estimated by dynamical modeling, and the mechanism for planet formation and the relevance of various planet atmosphere models are thereby understood.

Several of the exoplanets in Table 1 are highlighted elsewhere in this volume. Below we summarize the tentative new findings concerning Fomalhaut $b$.

\section{Fomalhaut $b$ Update}

At this conference I presented new observations with the Hubble Space Telescope showing the recovery of Fomalhaut $b$ in September, 2010. The most significant barrier in producing follow-up observations was that the coronagraphic camera that enabled the Fomalhaut $b$ discovery, the Advanced Camera for Surveys High Resolution Channel (ACS/HRC) suffered an electronics failure in January, 2007. The HST servicing mission in May 2009 restored function to the ACS Wide Field Channel (ACS/WFC), but the ACS/HRC was not successfully repaired. Meanwhile, adaptive optics imaging at nearinfrared wavelengths with the 10-m Keck telescope, as well as mid-infrared $(3.8 \mu \mathrm{m})$ imaging with the 8-m Gemini Observatory, lacked sufficient sensitivity to detect Fomalhaut $b$ from the ground. We therefore attempted to recover Fomalhaut b using direct imaging with the Wide Field Camera 3 infrared channel (WFC3/IR) using the F110W filter $(1.1 \mu \mathrm{m})$. The experiment failed due to previously unknown instrumental scattering spread over large azimuth angles and at a radius corresponding to the location of Fomalhaut $b$. We then studied the feasibility of using STIS coronagraphy or direct 
imaging with the ACS/WFC. Simulations showed that both cameras could be successful, but STIS should achieve a detection more efficiently than ACS/WFC (i.e. with a smaller allocation of telescope orbits). The details of the STIS observing strategy, data reduction and analysis will be presented elsewhere (Kalas et al. 2011).

Figure 1 summarizes our current findings. The new STIS observations show a point source near the expected 2010 location of Fomalhaut b with flux $0.4 \mu \mathrm{Jy}$, in agreement with the 2006 measurement (Kalas et al. 2008). The source location matches the expected position in the north-south direction, but lies approximately $0.15^{\prime \prime}$ west of the expected location for low eccentricity orbits. This discrepancy may indicate that Fomalhaut $b$ is on an eccentric orbit $(e \sim 0.67)$. However, we also expect uncorrected systematic errors arising from transforming between the ACS and STIS astrometric reference frames. The STIS observations were made in single guide star mode, increasing the uncertainty in the spacecraft roll angle. Also, our geometric distortion solution is derived from

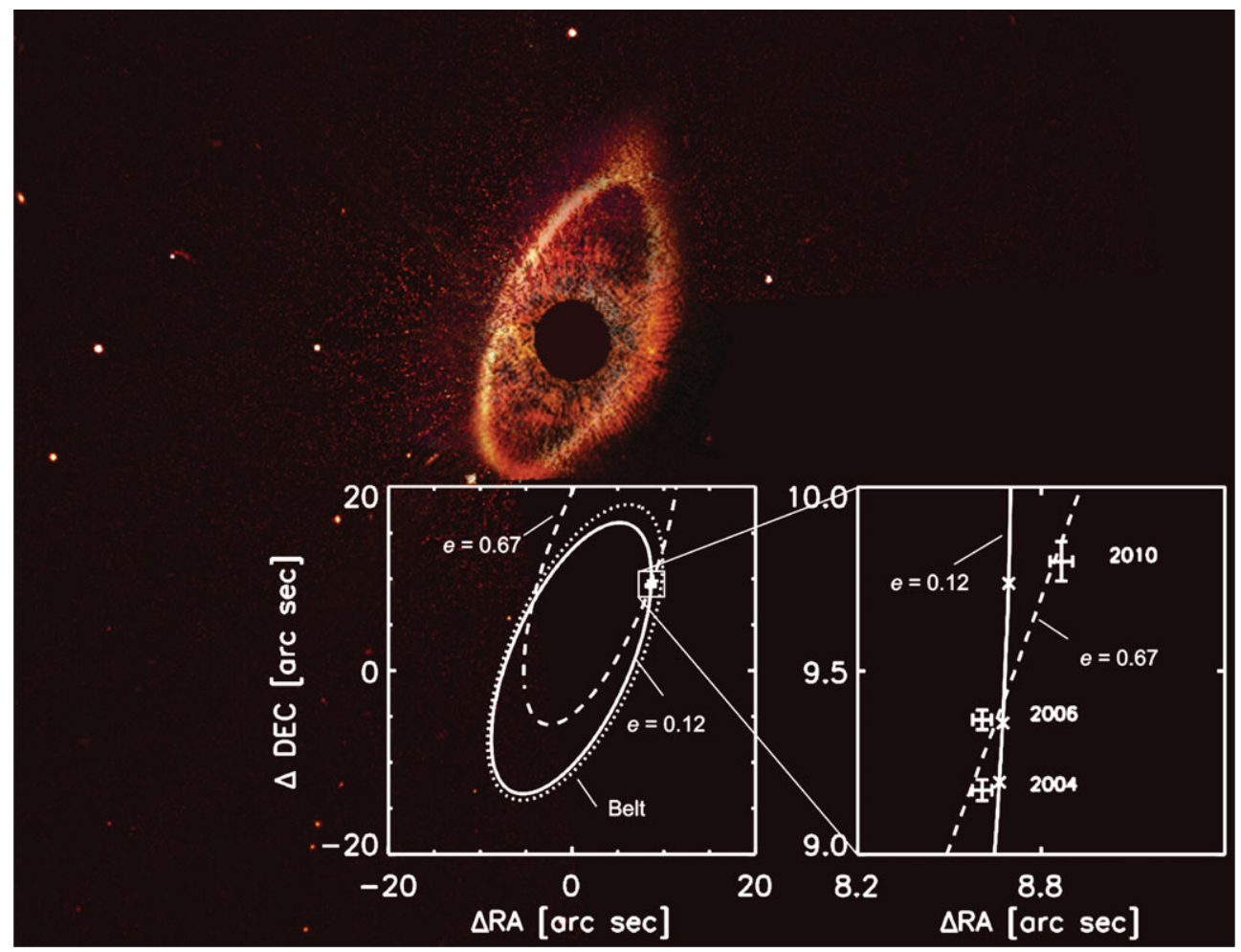

Figure 1. Composite image of the Fomalhaut system constructed from optical observations using three cameras aboard the Hubble Space Telescope (ACS/HRC, WFPC2, and STIS). The central star is occulted by coronagraphic spots. North is up, east is left. The tenuous halo north of the belt is a new feature discovered in recent STIS observations. The geometric offset of the belt $2^{\prime \prime}$ northward of the star is evident to the eye. The left bottom plot is drawn to the same scale as the image. The dotted line traces the inner edge of the belt, and the solid line traces an orbit nested within the belt with $e=0.12$. The right bottom plot magnifies our preliminary three-epoch $(2004,2006, \& 2010)$ astrometry and orbital fits. The ACS and STIS astrometric data are the three points with error bars, and the predicted positions are shown with the symbol $(\times)$ for the $e=0.12$ orbit. We are currently analyzing systematic errors due to uncorrected STIS distortion and a roll angle uncertainty due to the single guide star observation in 2010. At face value the observations are consistent with a best-fit bound orbit with $e=0.67$ that crosses the belt. We cannot rule out an unbound orbit until future observations are conducted. Monte Carlo simulations show that one additional observation in 2012 will exclude unbound orbits. 
calibration data obtained before the most recent servicing mission. Uncorrected distortions are evident in the calibrated STIS images because diffractions spikes have parabolic shapes. This uncertainty will be resolved when an updated STIS geometric distortion solution is available from new astrometric calibration observations.

Figure 1 also shows the discovery of a faint dust halo that extends northward approximately 4" beyond the previous outer boundary of the belt determined in the ACS/HRC data (Kalas et al. 2005). This finding more than doubles the measured extent of Fomalhauts dust belt. Two plausible explanations that are testable with multi-color imaging are: (1) the halo is due to small grains (grain size $a \sim \lambda / 2 \pi$ ) driven outward by radiation pressure, in which case the halo should be blue-scattering; or, (2) the eccentric orbit of Fomalhaut $b$ dynamically disturbs particles of all sizes, broadening the belt, as shown in dynamical simulations (Chiang et al. 2009), in which case the outer halo should share the scattered light color of the main belt.

Due to the uncertainties in the Fomalhaut $b$ astrometry, we currently cannot rule out the very intriguing possibility that it has a highly eccentric, belt-crossing orbit. This scenario implies that Fomalhaut $b$ may not be the object responsible for the secular perturbation that creates the 15 AU stellocentric belt offset (Kalas et al. 2005). Fomalhaut $c$ therefore remains to be discovered as the perturber. Furthermore, just as the majority of readers will witness $\beta$ Pic $b$ orbit its star over the next 16 years, Fomalhaut $b$ may offer the opportunity of witnessing a planet cross into its Kuiper Belt. Depending on the planet mass, belt crossings may need to be relatively fast to preserve the belt structure (e.g. orbital planes inclined relative to each other). Some type of periodic, close interaction with the belt may be consistent with the hypothesis that Fomalhaut $b$ is detected because of a large circumplanetary disk that is replenished over the age of the system.

\section{Circumplanetary disks}

Fomalhaut $b$ is unique in Table 1 because it is the only exoplanet detected in the optical. Kalas et al. (2008) report detections at 0.6 and $0.8 \mu \mathrm{m}$, with non-detections at $0.4,1.6$ and $3.8 \mu \mathrm{m}$. The $0.6 \mu \mathrm{m}$ flux is more than an order of magnitude greater than model atmospheres predict, suggesting non-thermal sources of optical emission. Another possibility is that the optical flux is light reflected from a circumplanetary ring. The non-detection at $0.4 \mu \mathrm{m}$ does not contradict this scenario due to the low signal-to-noise of observations made in this bandpass. Depending on factors related to the assumed geometry and albedo, the radius of the ring system is $20-40 R_{p}\left(R_{p} \sim 1.2 R_{J}\right)$. This would appear more like a circumplanetary disk than the main rings of Saturn, and the lack of a counterpart in our Solar System made this scenario for Fomalhaut $b$ appear somewhat speculative when we proposed it in 2008 .

As if on cue, Verbischer et al. (2009) reported that a counterpart for the circumplanetary disk hypothesis exists in the Solar System. The surface of Saturn's outer moon Phoebe ( $a=215 R_{p} ; R_{p} \sim 1.0 R_{J}$ ) is bombarded by micrometeoroids that launch fine dust in orbit around Saturn. The dust spirals inward due to radiation drag, forming a tenuous circumplanetary dust disk that is detected between $128-207 R_{p}$. Thus Saturn's largest ring is about five times larger than the ring postulated for Fomalhaut $b$. The estimated optical depth of the Phoebe ring is $10^{-8}$, but the inner Solar System at an age of 100 Myr had five to six orders of magnitude more interplanetary debris than the present epoch (Bottke et al. 2007 and references therein). Therefore an optically bright Phoebe ring at early times is entirely plausible.

However, Fomalhaut $b$ is not the only exoplanet where a puzzling spectrum evokes the existence of a circumplanetary disk. Near-infrared imaging and spectroscopy of 2M1207b 
give a model-dependent temperature of $1600 \mathrm{~K}$, but the luminosity of the planet is smaller than expected by an order of magnitude (Mohanty et al. 2007). An edge-on disk surrounding 2M1207b could produce significant gray extinction, or obscures the planet completely such that the flux received at Earth is due to photons scattered from the disk. A resolved example of such an optically thick case is HK Tau B. High angular resolution imaging shows that the source previously thought to be a direct image of a young protostar is actually the scattering surface of a disk, with the protostar completely occulted by the disk midplane (e.g., Koresko 1998). The main consequence of the circumplanetary disk hypothesis for 2M1207b is a very large uncertainty in its mass (Table 1).

Naturally the key difference between 2M1207b and Fomalhaut $b$ is that the latter has a significant source of external illumination from the host star, as in the case of Saturn's rings. For reference, Fomalhaut b is four times farther from its host star as Neptune is from the Sun, but the luminosity of Fomalhaut is a factor of $\sim 16$ greater than the Sun. Therefore, the incident stellar radiation flux on any circumplanetary material surrounding Fomalhaut b is roughly equivalent to the incident flux on Neptune.

One final note is the possibility that circumplanetary dust is produced from a system of irregular satellites, and thus the dust parent bodies reside in a cloud around the planet rather than a flattened disk. Kennedy \& Wyatt (2011) model the production of a dust cloud by irregular satellites surrounding Saturn. The resulting cloud has an hourglass morphology with apparent dimensions $2^{\circ} \times 1^{\circ}$ (for comparison the Moon's apparent diameter is $\left.0.5^{\circ}\right)$. The maximum radius of the cloud corresponds to approximately $1 / 2$ of a Hill radius $\left[R_{H}=a_{p}\left(1-e_{p}\right)\left(M_{p} / 3 M_{\star}\right)^{1 / 3}\right]$. If Fomalhaut b has $\mathrm{M}=1 \mathrm{M}_{J}$, then $1 / 2$ $R_{H} \sim 3$ AU. However, a dust cloud with diameter $6 \mathrm{AU}$ at the distance to Fomalhaut subtends $0.8^{\prime \prime}$ and would have been resolved with the HST observations. This constraint, as well as other considerations, particularly the collision lifetimes, suggest that Fomalhaut b may have $M<0.3 M_{J}$.

If the circumplanetary disk hypothesis is correct, orbital motion should change the viewing angles such that reflection and extinction vary significantly over time. Unfortunately, unlike Earth and Saturn analogs where the viewing angles change appreciably over months to decades (Arnold \& Schneider 2004), the orbital periods of Fomalhaut $b$ and 2M1207b approach a millennium. Nevertheless, secular variability in the optical to infrared spectra of exoplanets may eventually produce strong evidence for circumplanetary material around these two exoplanets and others.

\section{Summary}

The big picture is that 1.6 centuries after Neptune was discovered, we are once again finding and studying planets through direct imaging. If one simply tallies the planets in Table 1, we have just entered the domain of having more images of exoplanets than solar system planets. Moreover, two exoplanets may have giant ring systems that give us a notion of what Saturn or Jupiter may have resembled 4.5 billion years ago. Exoplanet imaging thus invokes a very powerful convergence of disciplines. From the study of planet atmospheres, to the theoretical framework of their formation, and proceeding to their dynamical evolution into long-lived systems, observation and theory will ultimately quantify the frequency and architectures of planetary systems.

\section{Acknowledgements}

I am grateful to James Graham (Dunlap Institute \& UC Berkeley) for the analysis of Fomalhaut b's astrometry and orbit, Michael Fitzgerald (UCLA) for significant work on ground-based observations of Beta Pic and Fomalhaut, as well as Mark Clampin (NASA GSFC) and Matt Mountain (STScI) for enabling the HST follow-up of Fomalhaut b. This 
work received support from the following: GO-11818 provided by NASA through a grant from STScI under NASA contract NAS5-26555; NSF AST-0909188; and the University of California LFRP-118057.

\section{References}

Arnold, L. \& Schneider, J. 2004, A\&3A, 420, 1123

Bottke, W. F., Levison, H. F., Nesvorny, D., \& Dones, L. 2007, Icarus, 190, 203

Bowler, B. P., Johnson, J. A., Marcy, G. W., et al. 2010, ApJ, 709, 396

Burrows, A. \& Liebert, J. 1993, Rev. Mod. Phys., 65, 301

Burrows, A., Sudarsky, D., \& Lunine, J. 2003, ApJ, 596, 587

Chauvin, G., Lagrange, A.-M., Dumas, C., et al. 2004, A\&A, 438, L25

Chauvin, G., Lagrange, A.-M., Dumas, C., et al. 2005, A\&A, 425, L29

Chauvin, G., Lagrange, A.-M., Bonavita, M., et al. 2010, A\&A, 509, A52

Chiang, E., Kite, E., Kalas, P., Graham, J. R., \& Clampin, M. 2009, ApJ, 693, 734

Ducourant, C., Teixeira, R., Chauvin, G., et al. 2008, A\&A, 477, L1

Dodson-Robinson, S. E., Veras, D., Ford, E. C., \& Beichman, C. A. 2009, ApJ, 707, 79

Duchene, G. 2008, New Astron. Revs, 52, 117

Fabrycky, D. C. \& Murray-Clay, R. A. 2010, ApJ, 710, 1408

Fortney, J. J., Marley, M. S., Saumon, D., \& Lodders, K. 2008, ApJ, 683, 1104

Galland, F., Lagrange, A.-M., Udry, S., et al. 2005, A\&A A, 443, 337

Kalas, P., Graham, J. R., \& Clampin, M. 2005, Nature, 435, 1067

Kalas, P., Graham, J. R., Chiang, E., et al. 2008, Science, 322, 1345

Kalas, P., Graham, J. R., Fitzgerald, M., \& Clampin, M. 2011, in prep

Kennedy, G. M. \& Wyatt, M. C. 2011, MNRAS, 412, 2137

Koresko, C. D. 1998, ApJ, 507, L145

Kratter, K. M., Murray-Clay, R. A., \& Youdin, A. N. 2010, ApJ, 710, 1375

Lafreniere, D., Doyon, R., Marois, C., et al. 2007, ApJ, 670, 1367

Lafreniere, D., Jayawardhana, R., \& van Kerkwijk, M. H. 2010, ApJ, 719, 497

Lagrange, A.-M., Gratadour, D., Chauvin, G., et al. 2009, A\&SA, 493, L21

Lucas, P. W., Weights, D. J., Roche, P. F., \& Riddick, F. C. 2006, MNRAS, 373, L60

Luhman, K. L. McLeod, K. K., \& Goldenson, N. 2005, ApJ, 623, 1141

Macintosh, B. A., Graham, J. R., Palmer, D. W., et al. 2008, SPIE, 7015, 31

Marley, M. S., Fortney, J. J., Hubickyj, O., Bodenheimer, P., \& Lissauer, J. J. 2007, ApJ, 655, 541

Marois, C., Macintosh, B., Barman, T., et al. 2008, Science, 322, 1348

Marois, C., Zuckerman, B., Konopacky, Q. M., et al. 2010, Nature, 468, 1080

Mohanty, S., Jayawardhana, R., Huelamo, N., \& Mamajek, E. 2007, ApJ, 657, 1064

Moro-Martin, A., Rieke, G. H., \& Su, K. Y. L. 2010, ApJ, 721, L199

Moya, A., Amado, P. J., Barrado, D., et al. 2010, MNRAS, 405, L81

Nero, D. \& Bjorkman, J. E. 2009, ApJ, 702, L163

Neuhauser, R., Guenther, E. W., Wuchterl, G., et al. 2005, A\&SA, 435, L13

Nielsen, E. L. \& Close, L. M. 2010, ApJ, 717, 878

Oppenheimer, B. R. \& Hinkley, S. 2009, ARAA, 47, 253

Rasio, A. F. \& Ford, E. B. 1996, Science, 274, 954

Raymond, S. N., Armitage, P. J., \& Gorelick, N. 2010, ApJ, 711, 772

Reidemeister, M., Krivov, A. V., Schmidt, T. O. B., et al. 2009, A\&A, 503, 247

Schmidt, T. O. B., Neuhauser, R., Seifahrt, A., et al. 2008, A\&EA, 491, 311

Seager, S. \& Deming, D. 2010, ARAA, 48, 631

Smith, B. A. \& Terrile, R. 1984, Science, 226, 1421

Su, K. Y. L., Rieke, G. H., Stapelfeldt, K. R., et al. 2009, ApJ, 705, 314

Veras, D., Crepp, J. R., \& Ford, E. B. 2009, ApJ, 696, 1600

Verbiscer, A. J., Skrutskie, M. F., \& Hamilton, D. P. 2009, Nature, 461, 1098

Zapatero Osorio, M. R., Bejar, V. J. S., Martin, E. L., et al. 2000, Science, 290, 103 